\title{
The overtraining reversal effect under two types of discrimination
}

\author{
VINCENT J. TEMPONE, JACK CAPEHART, GEORGE L, ATWOOD AND STEPHEN GOLDING
}

UNIVERSITY OF ARIZONA

The effects of overtraining were examined under both simultaneous and successive discrimination training. A significant overtraining reversal effect (ORE) was found. Further examination revealed a trend toward an ORE in the simultaneous condition which failed to reach significance and a significant ORE in the successive condition. Ss were 40, six to seven year old children. The ORE is examined in light of Lovejoy's (1966) mathematical model.

The present experiment was conducted to study possible differences in the overtraining reversal effect (ORE) as a function of whether the discrimination task is simultaneous or successive. None of the recent reviews (Paul, 1965; Mackintosh, 1965; Sperling, 1965) of the ORE deal specifically with the ORE in the successive discrimination. However, a recent mathematical theory by Lovejoy (1966) which is an extension of Mackintosh's (1965) attention theory would seem to make a differential prediction. One of the deductions from this theory is that the greater the difficulty of the initial discrimination the greater the magnitude of the ORE. Since it is generally found that the successive discrimination is more difficult than the simultaneous (Spence, 1952; Lipsitt, 1961; Loess \& Duncan, 1952) Lovejoy's theory would seem to predict a greater ORE in the successive discrimination. The present study was conducted to test this possibility.

\section{Method}

Apparatus. The apparatus was a vertically mounted display panel 35 in. $\times 36$ in. made from $1 / 2-$ in. plywood. Two stimulus apertures $8-1 / 4$ in. $x 10$ in. separated by a $1 / 2-$ in. woodstrip were centered on the display panel. Directly below each stimulus aperture and $2-1 / 2$ in. from the base of the display panel were two spring-held plungers that $S$ pulled to indicate his choice. At the lower left-hand side $4-1 / 2$ in. from the left side and 1-1/2 in. from the base was a slot 3 in. $x$ 1 in. through which reinforcers could be delivered into a small cup. The stimuli were black squares mounted on white cards. In the simultaneous group a 2 -in. square was paired with a 4 -in. square. During presentation the position of the large square relative to the small one varied from right to left. For the successive group the 2 -in. squares were paired in one setting, while two 4 -in. squares were paired in the second setting.

Procedure. Each child was run individually and sat facing the display board so that the stimulus cards appeared directly before him and the plungers were within easy reach. $\mathrm{S}$ was instructed that he was to play a game in which he would be shown cards with squares on them. As a card came into view, he was to choose one square or the other and then pull the plunger beneath. If correct, he would receive a marble; if incorrect, no marble would be given. The game was to see how soon $\mathrm{S}$ could get a marble every time. Following the instructions $\mathbf{E}$ presented the stimulus cards from behind the display board, recorded all responses, and delivered reinforcement for correct responses. The stimulus cards were presented to $S$ in a sequence preordered to guard against alternating and position strategies.

The experimental design incorporated two types of discrimination (simultaneous vs. successive) and two levels of training (criterion vs overtraining). Forty Ss with a mean age of six years nine months were subdivided into four groups of 10 each and were assigned to one of the four experimental groups. The simultaneous discrimination required $\mathrm{S}$ to choose between a large (4 in.) or a small (2 in.) square. In the successive discrimination $S$ had to respond either right or left when either two large squares or two small squares were presented. The correct or reinforced stimulus was alternated within each group. Ss in the criterion groups were required to get 9 correct responses out of any 10 successive trials. In the overtraining group the criterion was 18 correct responses out of any 20 successive trials. When $S$ met the respective criteria for the initial discrimination, reversal training was initiated. The criterion for successfully learning the reversal was 9 correct responses out of any 10 successive trials. Results

An analysis of variance was used to test the effects of the two types of discrimination training on learning the initial discrimination. Comparison between the simultaneous and successive groups was made using the number of trials to learn the initial discrimination to a criterion of 9 correct responses in any 10 successive trials. Using the above criterion the simultaneous group $(\bar{X}=20.45)$ learned the initial discrimination in significantly less trials than the successive group $(\bar{X}=$ 30.95); $\mathrm{F}=4.87, \mathrm{df}=1 / 38, \mathrm{p}<.05$.

Originally it was planned to use a 2 by 2 analysis of variance design to test the reversal effect; however, inspection of the data revealed a high degree of variability between the four experimental groups. Since an F max test showed this variance to be significant ( $F$ max $=75.74, \mathrm{df}=3 / 9, \mathrm{p}<.01)$ the Kruskall-Wallis test was used to test the effects of reversal training.

The mean number of trials to learn the reversal as a function of the type of discrimination and level of training are presented in Table 1. Table 1 also summarizes the comparisons made between the experimental groups. Although type of discrimination did not affect reversal training, there is a distinct trend which suggests that reversal is easier in the successive condition. The high degree of intersubject variability during reversal (Table 1) especially in the simultaneous criterion group probably obscured any difference between the two types of discriminations. The other main 
Table 1. Cells means and variances and Kruskall-Wallis analysis of the mean number of trials to learn a reversal discrimination for two types of discrimination and two levels of training.

\begin{tabular}{|c|c|c|c|c|}
\hline \multirow[t]{3}{*}{ Discrimination } & \multicolumn{4}{|c|}{ Troining } \\
\hline & \multicolumn{2}{|c|}{ Criterion } & \multicolumn{2}{|c|}{ Overtraining } \\
\hline & $\bar{x}$ & $s^{2}$ & $\bar{x}$ & $s^{2}$ \\
\hline Simultaneous & $27 . I$ & 720.30 & 20.7 & 232.45 \\
\hline Successive & 26.6 & 232.71 & 13.2 & 9.51 \\
\hline Comparison & & & $y$ & \\
\hline
\end{tabular}

Type of Discrimination

\begin{tabular}{lc} 
(Si vs Su) & .01 \\
evel of Training & \\
(C vs OT) & $5.47^{*}$ \\
Si C vs Si OT & .14 \\
Su C vs Su OT & $8.470^{* *}$ \\
Si C vs Su C & 2.20 \\
Si OT vs Su OT & 1.40 \\
\hline
\end{tabular}

$* p<.02$

$* * \quad p<.01$

variable, level of training, was significant. The ORE was obtained. Since the analysis of variance could not be used possible interaction effects between the main variables could not be examined. However, it was possible to examine the ORE under each type of discrimination. Such examination revealed a significant $O R E$ under the successive condition. In the simultaneous condition there is a trend that suggests a possible ORE but it failed to reach significance. Had more overtraining trials been used this trend might have been significant. Most theories of discrimination learning that have attempted to deal with the ORE would seem to predict a greater ORE in the simultaneous condition as opposed to the successive. The results of this study are in direct contradiction to such predictions.

\section{Discussion}

Lovejoy (1966) has proposed a two stage mathematical model which is compatible with the results of this study. Lovejoy attempts to explain the ORE by assuming that two stages are present in a discrimination task. The first stage consists of attending or not attending, while the second is a choice response. The model in this respect is similar to Mackintosh's (1965) two stage model. Lovejoy further assumes that the probability of choosing the correct response (PCR) increases at a greater rate than the probability of attending (PA). Thus, for Ss meeting the criteria of learning but not given overtraining PCR would be close to unity while PA although high would be some value less than unity. Therefore, the overtrained Ss would be more likely to be attending when they get their first reversal reinforcement. The non-overtrained Ss, however, would be more likely to get their first reversal reinforcement for non-attending.

Since the latter Ss have been rewarded for nonattending the probability of attending is decreased. The net effect would be that the non-overtrained Ss would be likely to stop attending to the relevant stimulus dimension while in the overtrained Ss, the probability of attending would be high during reversal. The model predicts that overtrained Ss would reverse faster.

of course, as already noted, many theories would predict the ORE. The unique feature of Lovejoy's model is that it assumes that when the relevant stimuli are very obvious, that the initial probability of attending to them is high. When PA is at asymptote both non-overtrained Ss and overtrained Ss would be "attending" when they receive their first reversal reinforcement. Thus, no ORE would be predicted. Using stat rats and varying $P A$ from .1 to 1.0 while holding the other parameters constant Lovejoy demonstrated that when the initial probability of attending PA is 1.0 no ORE is found; however, when PA varies from .3 to .7 an ORE is found.

Lovejoy then examined the empirical rat literature. When position has been the relevent dimension no ORE was usually found; however, with brightness as the relevant dimension an ORE was usually reported. Lovejoy argues that since a rat is a nocturnal animal, position is an obvious cue while brightness is less obvious.

Applying this model to the results of this study it seems reasonable that for a child the simultaneous discrimination is an easier or more obvious discrimination, while the successive discrimination is less obvious. The fact that in the initial discrimination the simultaneous was learned in less trials than the successive would support the above assertion. Similar results have been reported by Lipsitt (1961) using children and Loess \& Duncan (1952) with older human Ss. Thus in the simultaneous condition at the point of reversal PA is unity or close to unity in both criterion and overtrained Ss. Therefore, one does not find an ORE. In the successive condition, PA is lower initially. Since PCR increases at a greater rate than PA, at the point of reversal PA will be less than PCR in the criterion Ss while in the overtrained Ss PA will be approximately equal to PCR. Therefore, the probability of the "attending response" being reinforced during reversal is higher in the overtrained $S$ than in the nonovertrained Ss. Thus one would predict an ORE in the more difficult discrimination and no ORE in the easier or obvious discrimination. The results of this study conform to the predictions derived from Lovejoy's model.

\section{References}

Lipsitt, L. P. Simultaneous and successive discrimination learning in children. Child Develpm., 1961, 32, 337-347.

Loess, H. B., \& Duncan, C. P. Human discrimination learning with simultaneous and successive presentation of stimuli. J. exp. Psychol., 1952, 44, 215-221.

Lovejoy, E. Analysis of the overlearning reversal effect. Psychol. Rev., 1960, 73, 87-103.

Mackintosh, W. J. Selective attention in animal discrimination learning. Psychol. Bull., 1965, 64, 124-150.

Paul, C. Effects of overlearning upon single habit reversal in rats. Psychol. Bull., 1965, 63, 65-72.

Spence, $\mathbf{K}$. W. The nature of the response in discrimination learning. Psychol. Rev., 1952, 59, 89-93.

Sperling, Sally E. Reversal learning and resistance to extinction: a review of the rat literature. Psychol. Bull., 1965, 63, 281-297. 